\title{
A Performance Comparison Between Ad Hoc and Centrally Controlled CDMA Wireless LANs
}

\author{
Jeffrey Q. Bao, Member, IEEE, and Lang Tong, Senior Member, IEEE
}

\begin{abstract}
A performance comparison is presented between two types of code-division multiple-access wireless local area networks: centrally controlled and ad hoc networks. Based on a finite-population model, the network throughput, the average packet delay, and the network first exit time are derived for both systems. Two aspects of the performance comparison are addressed: 1) the comparison between the centrally controlled and the ad hoc architecture; and 2) the impact of spreading gain and error control coding on both systems. The efficiency of bandwidth utilization is investigated by normalizing the network performance with respect to the consumed bandwidth. Evaluations of these performance comparisons are also provided.
\end{abstract}

Index Terms-Ad hoc networks, code-division multiple-access (CDMA), performance analysis, slotted Aloha.

\section{INTRODUCTION}

W ITH THE proliferation of the Internet, there is an increasing demand for providing broadband wireless access to offices and homes. Wireless local area networks (LANs) require minimum infrastructure requirements and are becoming an attractive choice-of-technology for the emerging home and office networking market. A widely used architecture in wireless LAN, as shown in Fig. 1(a), is a network centrally controlled by the base station (BS) where every user communicates with others through the BS. An alternative is the ad hoc architecture shown in Fig. 1(b), where each user communicates with others directly. In this paper, we present a performance comparison between these two architectures.

Wireless LANs in applications such home/office networking often cover a small area with a relatively small number of nodes. This makes it reasonable to assume a one-hop topology that requires no dynamic reconfiguration. We will also restrict ourselves to packet-switched code-division multiple-access (CDMA) systems employing slotted Aloha random access protocols. ${ }^{1}$ Again, the restriction to a small coverage area allows us to ignore the near-far effect. We also assume that the spreading code of every node is known to every other node after initialization.

Manuscript received March 24, 2001; revised April 1, 2001; accepted August 1,2001 . The editor coordinating the review of this paper and approving it for publication is S. Tekinay. This work was supported in part by the Multidisciplinary University Research Initiative (MURI) under the Office of Naval Research under Contract N00014-00-1-0564 and in part by the Army Research Office under Grant ARO-DAAB19-00-1-0507.

J. Q. Bao is with the Broadband Networks Research Lab., Motorola Labs, Mansfield, MA 02048 USA (e-mail: Jeffrey.Q.Bao@motorola.com).

L. Tong is with the School of Electrical and Computer Engineering, Cornell University, Ithaca, NY 14853 USA (e-mail: 1tong@ee.cornell.edu).

Digital Object Identifier 10.1109/TWC.2002.805092

${ }^{1}$ If demand-assignment medium access control (MAC) protocols are used, the comparison here reflects only the random access part of the MAC.
A defining characteristic of CDMA is the possibility of receiving multiple packets at the same time. As demonstrated in this paper, the multipacket reception (MPR) capability of the network nodes can have a significant impact on the network performance, which affects the selection of network architecture. In contrast, for narrowband transmissions without using spatial and temporal diversity techniques, simultaneously transmitted packets are destroyed. Under this classical collision model, Aloha behaves the same in either a centrally controlled network or a fully connected ad hoc network.

The slotted Aloha protocol has been studied extensively. (See [1] for a collection of papers.) The work by Raychaudhuri [16] laid the foundation for the performance analysis of slotted Aloha CDMA systems, where simultaneously transmitted packets are assumed to have different spreading codes and are intended for different receivers. Pursley studied the performance of CDMA packet radio networks (the name for ad hoc systems in 1970s and 1980s) [15], [14] under the similar assumptions as that in [16]. A performance comparison between a slotted Aloha CDMA network and a multichannel narrowband slotted Aloha network can be found in [4], where the centrally controlled architecture with a transmitter-based CDMA scheme is assumed. When multiple transmitters transmit to the same receiver, CDMA systems can have the so-called "capture" effect which enables the receiver to recover the strongest or the earliest arrival packet out of multiple packets in a time slot. The effect of capture on the network performance for centrally controlled systems are investigated in [3], [5], and [11]. Polydoros and Silvester proposed a general framework [12] to incorporate the multiple-access capability and the capture effect into the analysis of slotted Aloha CDMA systems with dedicated transmitters and receivers.

While the behavior of the slotted ALOHA in a centrally controlled CDMA network is understood, little has been reported for ad hoc CDMA systems where nodes transmit directly to each other and any node can be a potential transmitter or receiver. One reason, as suggested in [17], is that the analysis becomes intractable due to the uncoordinated behavior of nodes in such networks. Therefore, the effect of the ad hoc architecture on the network performance has not been investigated. Furthermore, the effects of spreading gain and error control coding on the network performance are not fully understood. In this paper, we present analysis that overcomes this difficulty.

The contribution of this paper is as follows: 1) the performance of ad hoc systems employing transmitter-based CDMA scheme is analyzed;2) the effect of the network architecture on the network performance is studied by comparing the performance of an ad hoc system with that of a centrally controlled system; and 3) impacts of the spreading gain and error control 


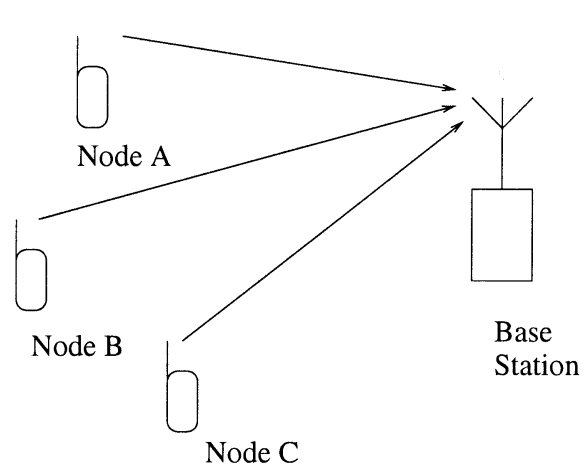

(a)

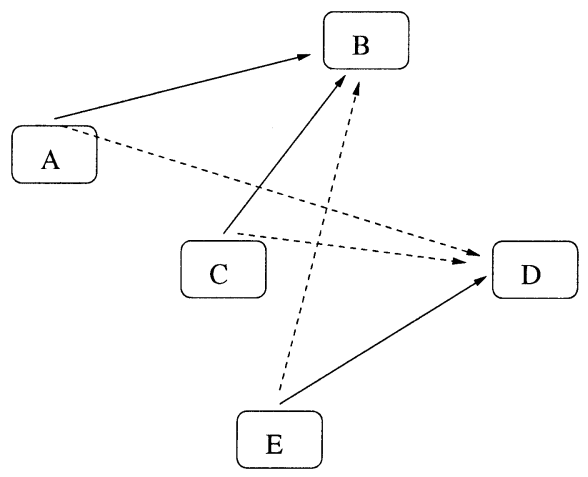

(b)

Fig. 1. (a) Centrally controlled system. (b) Ad hoc system.

coding on the network performance of both systems, which also lead to the understanding of efficiency of bandwidth utilization in both systems, are investigated. Specifically, based on a finite-population model, the network throughput, average packet delay and the first exit time (FET) of the two systems are derived and performance comparisons are evaluated. Furthermore, effects of spreading gain and error control coding on the network performance of both systems are quantitatively analyzed.

The paper is organized as follows. In Section II, we provide models of these two networks and necessary assumptions for the analysis. Section III characterizes the Markov chain modeling of these two systems. We devote Sections IV, V, and VI to the performance comparison of the network throughput, average packet delay, and the network stability, respectively.

\section{Two System ModELS}

We consider a packet-switched CDMA network using the slotted Aloha random access protocol under two different network architectures: the centrally controlled network and the ad hoc network. First, we describe the models of these two systems, then, we present necessary assumptions for the performance analysis.

\section{A. System Descriptions}

Centrally Controlled Network: The first system is the centrally controlled system as shown in Fig. 1(a). This is equivalent to a cellular network with a single cell. In this paper, the term "cellular" network is used synonymously with the "centrally controlled" network. In this architecture, multiple nodes transmit packets to each other through the BS, i.e., nodes transmit packets to a BS via the uplink and the BS relays these packets to potential receiving nodes through the downlink. We assume a time division duplex (TDD) system with equal-sized uplink and downlink packets, each occupies one time slot. Nodes are half-duplex and are always in the receiving mode during the downlink period. During the uplink period, nodes are in the transmitting mode. A slotted Aloha random access protocol is used by all nodes in the uplink: whenever a node has a new packet to transmit, it sends the packet in the earliest available uplink time slot. If the packet is not successfully received by the $\mathrm{BS}$, the node will retransmit the packet with a fixed probability in each successive uplink slots until a successful transmission occurs.

Each node in the network transmits packets in the uplink using an unique spreading code which is assumed to be randomly generated. The BS has the knowledge of each node's code. We assume that the receiver at the BS is a bank of matched filters. Furthermore, we assume that in the downlink the BS uses orthogonal codes for packets intended for different nodes so that each receiving node always successfully receives its packets and the transmission success of a packet depends on the uplink reception alone.

Ad Hoc System: Fig. 1(b) illustrates an ad hoc network. Nodes transmit to each other directly through a common channel by which all nodes are fully connected. Each node can be a transmitter or receiver. The same slotted Aloha random access protocol used in the centrally controlled network is also employed by all nodes. The transceiver at each node is also half-duplex [15]. Every node uses a unique code to spread its transmitted packets. In order to receive packets from any potential nodes, we assume that each node has the knowledge of all possible spreading codes and the receiver at each node is also a bank of matched filters.

While the multiple packet reception (MPR) capability of a node due to the spread-spectrum modulation in the ad hoc network is the same as that of the BS in the centrally controlled network, the reception capability of nodes in the two systems are fundamentally different. For example, because transceivers are half-duplex, a transmitting node in the ad hoc network cannot receive packets from other nodes. One can view this as a "collision" between a transmitter and a receiver. In a centrally controlled network, in contrast, only transmitters can collide among each other.

\section{B. Assumptions and Notations}

We follow the convention used in the classical analysis of slotted Aloha by Kleinrock and Lam [8]: a node which needs to retransmit a packet is referred to as in the backlogged state; otherwise a node is in the unbacklogged state. We adopt the finite population model, i.e., the total number of nodes in both systems is finite. To simplify analysis, we ignore noises and assume that errors in a packet are caused by multiple-access interference (MAI) alone. A linear block code is used for error correction. We make the following five assumptions about both 
TABLE I

Key PARAMETERS AND NOTATIONS

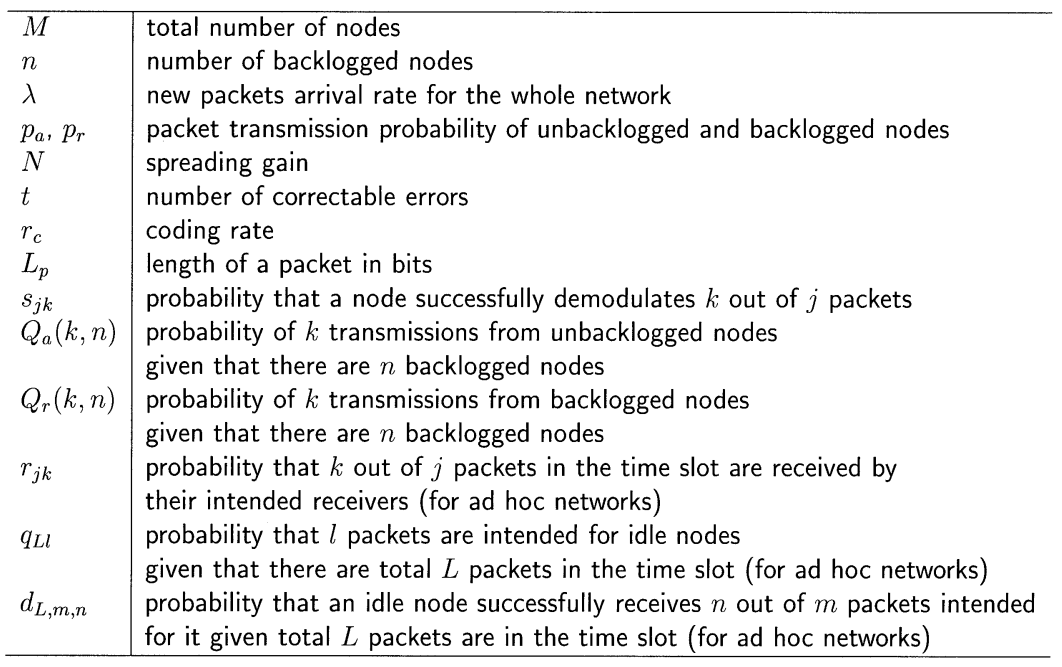

the centrally controlled and the ad hoc systems with key notations listed in Table I.

Assumption 1: Nodes generate packets according to independent Poisson processes with equal arrival rate.

Assumption 2: There is an immediate feedback about the status of the transmission.

Assumption 3: There is no buffer at any node, i.e., each node can at most hold one packet at a time.

Assumption 4: With probability $s_{k i}$, the receiver at the BS or a node in the ad hoc system detects successfully $i$ out of $k$ colliding packets in a time slot.

Assumption 5: Each node has equal probability to transmit to every other node.

Note that Assumption 1-Assumption 3 are standard assumptions for the analysis of slotted Aloha systems with finite number of nodes [2]. Assumption 4 is due to the matched filter receiver structure described previously. Further, note that Assumption 4 implies the perfect power control, which is approximately valid for LANs. This assumption enables us to obtain analytical expressions of the network performance which can provide insights into the behavior of ad hoc CDMA systems.

\section{MARKOVIAN ANALYSIS}

We follow the Markov chain approach proposed by Kleinrock and Lam [8] for the finite-population model analysis with the number of backlogged nodes $n$ as the network state. For an $M$-node network, the Markov chain is characterized by the $(M+1) \times(M+1)$ transition matrix $P=\left[p_{n k}\right]$ with $p_{n k}$ being the probability that the network state goes from $n$ to $k$ in one transition. Next, we characterize the Markov chain for both systems by obtaining the transition matrix of each system.

\section{A. Characterizations of the Centrally Controlled Network}

In the centrally controlled system, the network state changes every two time slots (packets are transmitted during the uplink time slot and received in the downlink time slot), therefore, the transition probability $p_{n k}$ is the probability that the network state goes from $n$ to $k$ in two time slots. To obtain the state tran- sition matrix $P_{c}=\left[p_{n k}^{c}\right]$, we first define the reception matrix $S$ for the BS

$$
S=\left(\begin{array}{ccccc}
s_{10} & s_{11} & 0 & \cdots & 0 \\
s_{20} & s_{21} & s_{22} & \cdots & 0 \\
\vdots & & & & \vdots \\
s_{M 0} & s_{M 1} & s_{M 2} & \cdots & s_{M M}
\end{array}\right)
$$

where $s_{j k}$ is the probability that the BS successfully demodulates $k$ out of $j$ packets.

Elements of $S$ are a function of the probability of a node to successfully detect a packet in a collision. For CDMA packet systems, given total number of packets in a slot, it is difficult to determine the exact probability of successfully detecting a packet by a receiver. Even if we ignore channel noise, evaluating bit-error rate (BER) is a nontrivial task [9]. Furthermore, bit errors in a packet do not occur independently [6]. Since we do not intend to study how to obtain a more accurate approximation of packet success probability, we use a standard Gaussian assumption provided in [6] about the MAI, i.e., the output of a matched filter corresponding to MAI components is assumed to be a white Gaussian random process. Furthermore, we assume bit errors happen independently within a packet.

The computation of packet success probability also follows [6]. Let $k$ be the total number of packets in a slot, $N$ be the spreading gain, and $z$ be the output of each correlator corresponding to the MAI components. In the standard Gaussian assumption, $z$ is assumed to be a white Gaussian random process. The variance of $z$ is given in [9] to be $(k-1)(N / 3)$. The BER $x$ is given by

$$
x=Q\left(\sqrt{\frac{3 N}{k-1}}\right)
$$

where $Q(y)=(1 / \sqrt{2 \pi}) \int_{y}^{\infty} e^{-\left(t^{2} / 2\right)} d t$. Under the assumption that errors occur independently in a packet, we then have the packet success probability

$$
p_{c}(k)=\sum_{i=0}^{t}\left(\begin{array}{c}
L \\
i
\end{array}\right) x^{i}(1-x)^{L_{p}-i}
$$


where $t$ is the number of bit errors that can be corrected by coding. If each matched filter works independently at the BSs receiver, $s_{n k}$ is the probability that $n$ out of $k$ independent Bernoulli trials are successful with single trial success probability $p_{c}(k)$, hence, we have

$$
s_{k n}=\left(\begin{array}{c}
k \\
n
\end{array}\right) p_{c}(k)^{n}\left(1-p_{c}(k)\right)^{k-n} .
$$

Following [16] and utilizing the fact that the change of the network state is determined by the difference between the number of unsuccessful transmissions from unbacklogged nodes and the number of successful transmissions from backlogged nodes, we can obtain $p_{n k}^{c}$ as follows: let $Q_{a}^{c}(k, n)$ be the probability that $k$ unbacklogged nodes transmit packets in a given uplink slot and $Q_{r}^{c}$ the probability that $k$ backlogged nodes transmit

$$
Q_{a}^{c}(k, n)=\left(\begin{array}{c}
M-n \\
k
\end{array}\right)\left(1-p_{a}^{c}\right)^{M-n-k}\left(p_{a}^{c}\right)^{k}
$$

and

$$
Q_{r}^{c}(k, n)=\left(\begin{array}{c}
n \\
k
\end{array}\right)\left(1-p_{r}\right)^{n-k} p_{r}^{k}
$$

where $p_{a}^{c}=1-e^{-(2 \lambda / M)}$ is the the probability that there is at least one packet arrives at an unbacklogged node during two slots for the Poisson arrival with rate $\lambda$ and $p_{r}$ is the retransmission probability for a backlogged node during the uplink slot. The transition probability $p_{n k}^{c}$ is given by (7) shown at the bottom of the page with $s_{00}$ defined to be one. Since the Markov chain defined is irreducible and aperiodic, the stationary distribution of the network state $\left\{q^{c}\right\}_{n=0}^{M}$ can be obtained by solving the following balance equation:

$$
\overrightarrow{q^{c}}=\overrightarrow{q^{c}} P_{c}
$$

where $\overrightarrow{q^{c}}=\left[q_{0}^{c}, q_{1}^{c}, \ldots, q_{M}^{c}\right]$ and $\sum q_{n}^{c}=1$.

\section{B. Characterizations of the Ad Hoc Network}

Since the network state can change during one time slot in the ad hoc network, the transition probability $p_{n k}$ is defined for every time slot. In the centrally controlled system, the Markov chain transition matrix only depends on $s_{n k}$ which is related to the receiver capability. In the ad hoc system, however, determining the Markov chain transition matrix is not straightforward. The reason is that $s_{n k}$ does not completely characterize the multiple packet reception capability of the network. For example, in Fig. 2 node A and B are transmitting, node C is receiving. A solid line indicates the packet is intended for a node, whereas a dotted line indicates the packet is not intended for the node but is also presented at the receiver because of the full connectivity. Clearly, node B cannot receive node A's packet due to half-duplex operation of its transceiver. Furthermore, if node C

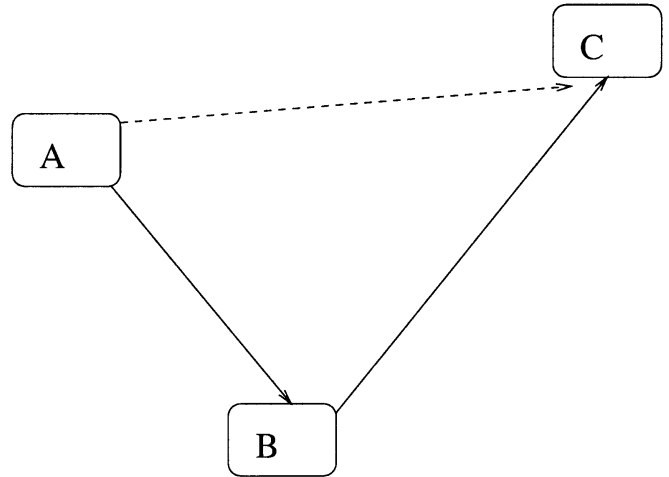

Fig. 2. Two issues in DS/SS slotted Aloha ad hoc networks.

successfully detects one packets, there is only half chance that the packet from B's is detected. To take into account the node availability and the possibility that a node detects packets not intended for it, we define the network reception matrix $R$ as

$$
R=\left(\begin{array}{ccccc}
r_{10} & r_{11} & 0 & \cdots & 0 \\
r_{20} & r_{21} & r_{22} & \cdots & 0 \\
\vdots & & & & \vdots \\
r_{M 0} & r_{M 1} & r_{M 2} & \cdots & r_{M M}
\end{array}\right)
$$

where $r_{j k}$ is the probability that $k$ out of $j$ packets in the time slot are received by their intended receivers in the network.

In general, $R$ is a function of $S$ and the network traffic pattern. The conversion of $S$ to $R$ in an ad hoc system is provided by the following theorem.

Theorem 1: Under Assumption 1-Assumption 5, given total $L \leq M$ packets are transmitted in a time slot, the probability that there are $n \leq L$ successfully received packets by their intended receivers in the network is given by

$$
\begin{aligned}
& r_{L n}=\sum_{l=n}^{L} \sum_{J=\min (l, 1)}^{\min (l, M-L)} q_{L l} \frac{\left(\begin{array}{c}
M-L \\
J
\end{array}\right)}{(M-L)^{l}} \\
& \times \sum_{\sum_{j=1}^{J} a_{j}=l} \frac{l !}{a_{1} ! a_{2} ! \ldots a_{J} !} \times\left(\sum_{\sum_{j=1}^{J} b_{j}=n} \prod_{i=1}^{J} d_{L, a_{i}, b_{j}}\right)
\end{aligned}
$$

where $a_{j}=1,2 \ldots l, b_{j}=0,1, \ldots a_{j}$ and

$$
\begin{aligned}
q_{L l} & =\left(\begin{array}{c}
L \\
l
\end{array}\right)\left(\frac{M-L}{M-1}\right)^{l}\left(\frac{L-1}{M-1}\right)^{L-l} \\
d_{L, a_{i}, b_{i}} & =\sum_{k=b_{i}}^{L-\left(a_{i}-b_{i}\right)} \frac{\left(\begin{array}{c}
a_{i} \\
b_{i}
\end{array}\right)\left(\begin{array}{l}
L-a_{i} \\
k-b_{i}
\end{array}\right)}{\left(\begin{array}{l}
L \\
k
\end{array}\right)} s_{L k} .
\end{aligned}
$$

A proof is provided in Appendix A.

$$
p_{n k}^{c}= \begin{cases}\sum_{y=n-k}^{n} \sum_{x=0}^{M-n} s_{(x+y)[x+(n-k)]} Q_{r}^{c}(y, n) Q_{a}^{c}(x, n), & 0 \leq k<n \\ \sum_{x=k-n}^{M-n} \sum_{y=0}^{n} s_{(x+y)[x-(k-n)]} Q_{a}^{c}(x, n) Q_{r}^{c}(y, n), & n \leq k \leq M\end{cases}
$$


Once we have $R$, we can substitute $s_{i j}$ by $r_{i j}$ into (7) and obtain the Markov chain transition matrix of the ad hoc network $P_{a}=\left[p_{n k}^{a}\right]$ by (13) at the bottom of the page, where

$$
Q_{a}^{a}(k, n)=\left(\begin{array}{c}
M-n \\
k
\end{array}\right)\left(1-p_{a}^{a}\right)^{M-n-k}\left(p_{a}^{a}\right)^{k}
$$

and

$$
Q_{r}^{a}(k, n)=\left(\begin{array}{l}
n \\
k
\end{array}\right)\left(1-p_{r}\right)^{n-k} p_{r}^{k}
$$

are probabilities that $k$ packets are transmitted by unbacklogged and backlogged nodes in one time slot, respectively, and $p_{a}^{a}$ and $p_{r}$ are packet transmission probabilities for unbacklogged and backlogged nodes in one time slot in the ad hoc system, respectively, $r_{00}$ is also defined to be one. The Markov chain is also irreducible and aperiodic. Note the difference between $p_{a}^{a}$ and $p_{a}^{c}: p_{a}^{a}$ is the probability that an unbacklogged node transmits a packet in one time slot and is given by $p_{a}^{a}=1-e^{-(\lambda / M)}$.

Similar to the centrally controlled system, the stationary distribution $\left\{q^{a}\right\}_{n=0}^{M}$ of the ad hoc system can be obtained by solving the Markov-chain balance equation

$$
\overrightarrow{q^{a}}=\overrightarrow{q^{a}} P_{a}
$$

where

$$
\overrightarrow{q^{a}}=\left[q_{0}^{a}, q_{1}^{a}, \ldots, q_{M}^{a}\right]
$$

and

$$
\sum q_{n}^{a}=1
$$

Next, we proceed to compare the performance of the centrally controlled and the ad hoc system.

\section{THROUGHPUT COMPARISON}

The network throughput is defined as the average number of packets successfully received by their intended receivers in a time slot. We can apply classical results [2] to obtain formulae for the throughput for both systems.

Throughput of the Centrally Controlled Network: Given network state $n$, the number of packets successfully received by their intended receivers in two time slots is

$$
\tilde{N}=\sum_{k=1}^{M} p_{k}^{c} \sum_{l=0}^{k} l s_{k l}
$$

where

$$
p_{k}^{c}=\sum_{x=0}^{k} Q_{a}^{c}(x, n) Q_{r}^{c}(k-x, n)
$$

is the probability that total $k$ packets are transmitted in the uplink time slot. Because the throughput $\beta_{\mathrm{c}}(n)$ and the average throughput $\bar{\beta}_{\mathrm{c}}$ is defined per time slot, we can obtain them by the following:

$$
\begin{aligned}
\beta_{\mathrm{c}}(n) & =\frac{\tilde{N}}{2} \\
\bar{\beta}_{c} & =E\left(\beta_{c}(n)\right)=\sum_{n=0}^{M} \beta_{c}(n) q_{n}^{c}
\end{aligned}
$$

where $q_{n}^{c}$ is the stationary distribution of the network state Markov chain.

Throughput of the Ad Hoc Network: Similarly, we can have the throughput $\beta_{a}(n)$ and the average throughput $\bar{\beta}_{a}$ of the ad hoc system

$$
\begin{aligned}
\beta_{a}(n) & =\sum_{k=1}^{M} p_{k}^{a} \sum_{l=0}^{k} l r_{k l} \\
\bar{\beta}_{a} & =E\left(\beta_{a}(n)\right) \\
& =\sum_{n=0}^{M} \beta_{a}(n) q_{n}^{a}
\end{aligned}
$$

where $p_{k}^{a}$ is the probability that total $k$ packets are transmitted in one time slot in the ad hoc network.

\section{A. Throughput Bound}

When we have the perfect receiver, i.e., all collided packets can be received successfully by a receiver, intuition suggests that the throughput should reach the maximum. Indeed, for centrally controlled systems, when

$$
s_{j k}=\left\{\begin{array}{ll}
1, & j=k \\
0, & j \neq k
\end{array} \quad \text { for } \quad j=1,2, \ldots, M\right.
$$

every transmitted packets will be received correctly by the BS, therefore, the throughput is equal to the arrival traffic. However, for the ad hoc system, the architecture and the half-duplex mode of transceivers impose limits on the throughput. The following theorem provides the performance bounds of an ad hoc system. The proof can be found in Appendix B.

Theorem 2: Under Assumption 1-Assumption 5

$$
\begin{aligned}
\beta_{a}(n) & \leq \sum_{k=1}^{M} p_{k} \sum_{l=0}^{k} l q_{k l} \\
& =\sum_{k=1}^{M} p_{k} \sum_{l=0}^{k} l\left(\begin{array}{c}
k \\
l
\end{array}\right)\left(\frac{M-L}{M-1}\right)^{l}\left(\frac{L-1}{M-1}\right)^{k-l} .
\end{aligned}
$$

The equality holds iff $S=S_{*}$, where

$$
S_{*}=\left[\begin{array}{ccccc}
0 & 1 & 0 & \cdots & 0 \\
0 & 0 & 1 & \cdots & 0 \\
\cdots & & \cdots & & \cdots \\
0 & 0 & 0 & \cdots & 1
\end{array}\right] .
$$

$$
p_{n k}^{a}= \begin{cases}\sum_{y=n-k}^{n} \sum_{x=0}^{M-n} r_{(x+y)[x+(n-k)]} Q_{r}^{a}(y, n) Q_{a}^{a}(x, n), & 0 \leq k<n \\ \sum_{x=k-n}^{M-n} \sum_{y=0}^{n} r_{(x+y)[x-(k-n)]} Q_{a}^{a}(x, n) Q_{r}^{a}(y, n), & n \leq k \leq M\end{cases}
$$



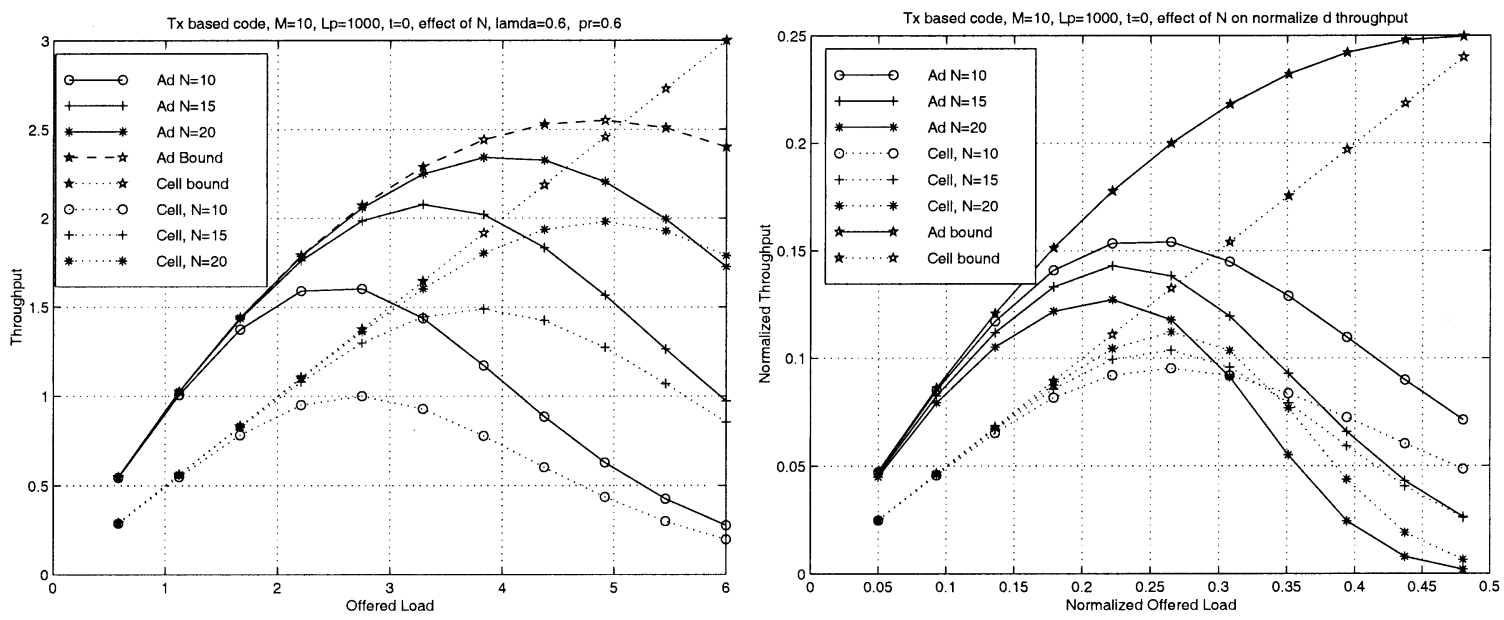

Fig. 3. Throughput versus offered load for $t=0 \lambda=0.6, p_{r}=0.6$. Solid line: ad hoc networks; dotted line: centrally controlled networks; left: actual; right: normalized.

\section{B. Normalization of the Throughput}

Because the throughput is a function of network resources such as the bandwidth, to compare fairly the throughput of systems with different bandwidth requires a normalization. We define the normalized throughput as the average number of information bits successfully received by their intended receivers per second per hertz [12]. Assuming binary phase-shift keying (BPSK) modulation, given the network throughput $\beta$, spreading gain $N$, length of the packet $L_{p}$, coding rate $r_{c}$, and symbol duration $T_{s}$, the average number of successfully transmitted information bits per slot is $L_{p} r_{c} \beta$; duration of each time slot is $L_{p} T_{s}$ and the consumed bandwidth is $N\left(1 / T_{s}\right)$. Therefore, we have the normalized throughput $\beta_{u}$

$$
\beta_{u}=\frac{L_{p} r_{c} \beta}{L_{p} T_{s} N \frac{1}{T_{s}}}=\frac{r_{c} \beta}{N} .
$$

Spreading gain $N$ is a design parameter that can be varied. To determine coding rate $r_{c}$, we apply the Gilber-Varsharmov lower bound for block codes [13]. Following [7], given packet length $L_{p}$ and number of correctable errors $t$, we can have the maximum coding rate by the following two steps:

$$
\begin{aligned}
\alpha & =\frac{2 t+1}{L_{p}} \\
r_{c} & =1+\alpha \log _{2}(\alpha)+(1-\alpha) \log _{2}(1-\alpha) .
\end{aligned}
$$

The normalization in (23) applies to both throughput and average throughput. Given the total number of nodes $M$ to achieve the perfect receiver $S_{*}$, we can let the spreading gain to be $M$ and assign each node one of the $M$ orthogonal codes. Therefore, we can let $N=M$ and $r_{c}=1$, then apply (23) to obtain the normalized maximum throughput.

\section{Throughput Comparison Evaluation}

In the following throughput comparisons, we assumed $M=$ 10 nodes in both the centrally controlled (cellular) and the ad hoc system, each packet is 1000-b long (i.e., $L_{p}=1000$ ). Two types of comparison are presented. First, we compared the throughput performance between the centrally controlled and the ad hoc network under the same physical parameters (i.e., same $N$ and $t$ ). Second, we investigated the effects of $N$ and $t$ on the throughput for both systems.

Comparison between the centrally controlled and the ad hoc system: We evaluated throughput of both the centrally controlled (cellular) and the ad hoc systems with varying $N$ at a particular $t$. Figs. 3-5 shows both the actual throughput versus offered load and normalized throughput versus normalized offered load (left: actual, right: normalized) for these two systems with varying error correction capability $(t=0,5,10$, respectively).

We observed from Figs. 4 and 5 that with a moderately powerful receiver (spreading gain $N>10$ and number of correctable bit errors $t>0$ ), the ad hoc system had higher throughput than that of the centrally controlled system under light traffic conditions (offered load $<4.5$ ); but under heavy traffic conditions (offered load $>4.5$ ), the centrally controlled system out-performed the ad hoc system. The reason is that under light traffic conditions and with a moderate powerful receiver, most of the transmitted packets were successfully received by either the BS in the centrally controlled network or by intended nodes in the ad hoc network, but in the centrally controlled system, these successfully received packets had to be transmitted again by the BS to their intended receivers during the downlink slot. Although we assume that these packets are never lost on the downlink, the throughput is only half of the number of successfully received packets by the BS. Under heavy traffic conditions, however, the performance bound of the ad hoc system limits the throughput; while in the centrally controlled system, there is no such throughput bound and the throughput can be higher than that of the ad hoc system. In centrally controlled systems with a poor performance receiver (see Fig. 3 with $t=0$ ), the advantage of no throughput bound cannot show up because under heavy traffic conditions, most of the packets cannot be received by the BS. Hence, the ad hoc system still out-performed the centrally controlled system.

Another interesting observation from Figs. $3-5$ is that with the same spreading gain and error control coding, the offered load that maximizes the throughput was higher in the centrally 

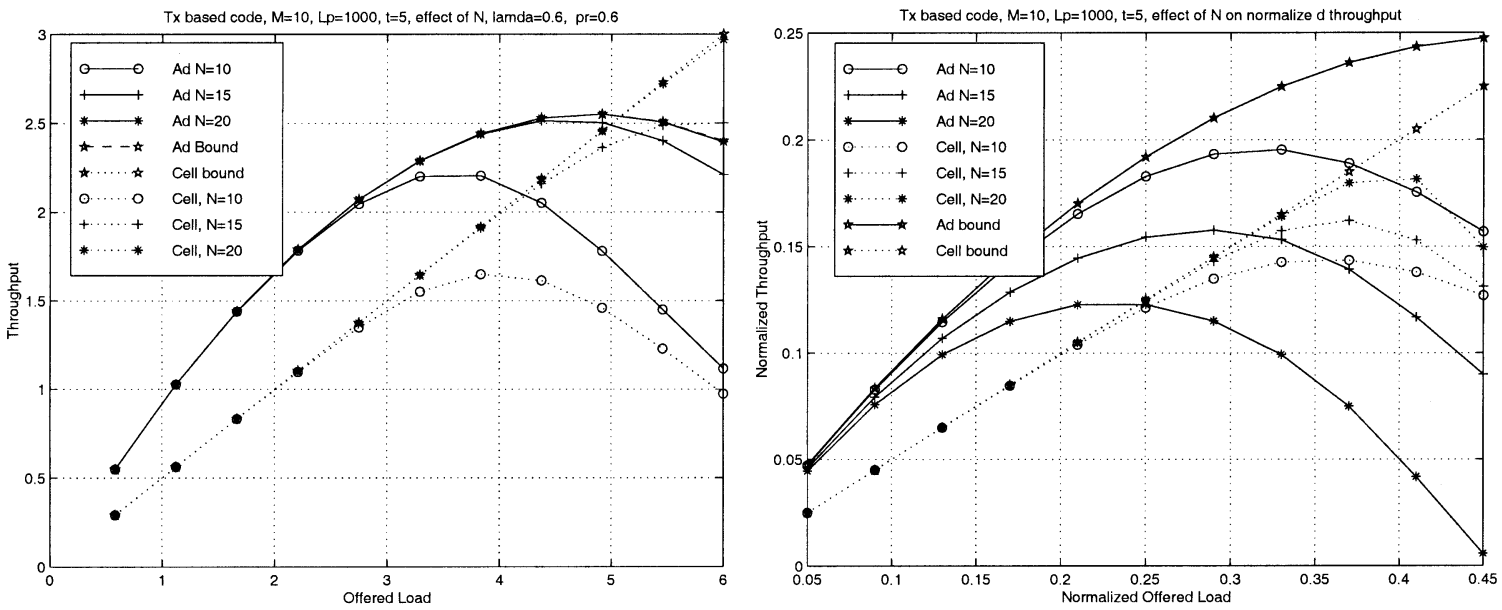

Fig. 4. Throughput versus offered load for $t=5 \lambda=0.6, p_{r}=0.6$. Solid line: ad hoc networks; dotted line: centrally controlled networks; left: actual; right: normalized.
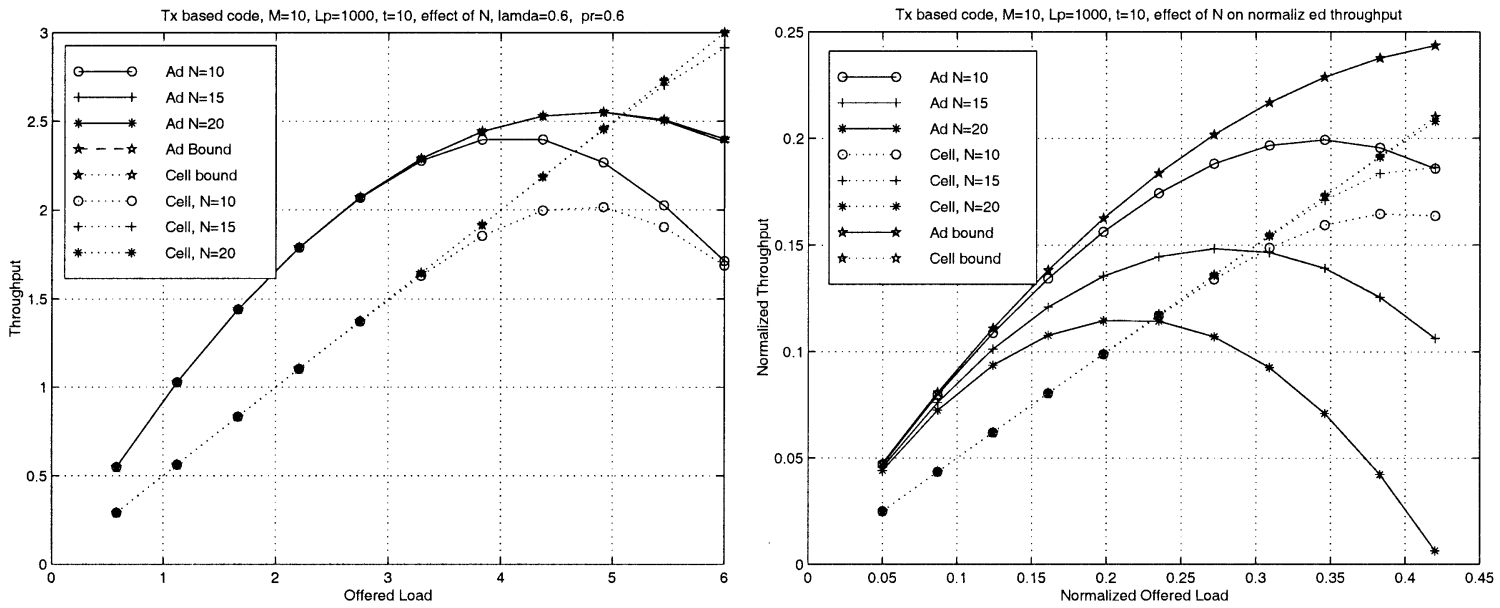

Fig. 5. Throughput versus offered load for $t=10 \lambda=0.6, p_{r}=0.6$. Solid line: ad hoc networks; dotted line: centrally controlled networks; left: actual; right: normalized.

controlled network than in the ad hoc network (for example, in Fig. 4 with $N=15$ and $t=5$, the centrally controlled system reached the maximum throughput when offered load was five; but in the ad hoc network, the maximum throughput was achieved at around 4.4. This behavior, again, is due to the throughput bound of the ad hoc system. As the traffic became heavier, packets were more likely targeted for busy nodes and the possibility of a node detecting packets intended for other nodes increased in the ad hoc network. Even a node is still capable of detecting packets successfully, the throughput of the ad hoc system started to decrease, but in the centrally controlled system, the throughput began to decrease only after the traffic was beyond the BSs capability. Therefore, the centrally controlled system could reach the maximum of the throughput at heavier offered load than the ad hoc system could.

Effects of Spreading Gain on the Throughput: For both systems, receivers should have higher probability to successfully detect packets for larger $N$ because larger $N$ leads to more orthogonal codes, hence, less MAI. In the centrally controlled system, the actual throughput approached half of the offered load as $N$ increased. For example, as shown in Fig. 5, with $t=10$ and $N=20$, the BS recovered almost all packets in a collision. Therefore, the throughput was almost half of the offered load at all load conditions. However, for the ad hoc system, as indicated by Theorem 2, the actual throughput was only close to the performance bound as shown in Figs. 4 and 5.

Although it is intuitive that a higher spreading gain $(N)$ will lead to higher actual throughput, the effect of $N$ on normalized throughput is not obvious. We observed from Figs. 3-5 that in centrally controlled systems, larger $N$ indeed lead to higher normalized throughput except when $t=0$ and normalized throughput was greater than 0.35 . For ad hoc systems, in contrast, larger $N$ actually lead to smaller normalized throughput. Apparently, in the centrally controlled system, the throughput improvement was large enough to offset the bandwidth expansion introduced by the increased spreading gain before the normalized offered load reached certain threshold after which the bandwidth expansion became the dominant factor in determining the normalized throughput (see Fig. 3). We can also observe that as the receiver became more powerful ( $t$ increased), the threshold after which the normalized throughput began to decrease for larger $N$ became higher. In the ad hoc system, the bandwidth expansion cannot overcome the limitation on the throughput imposed by the node 

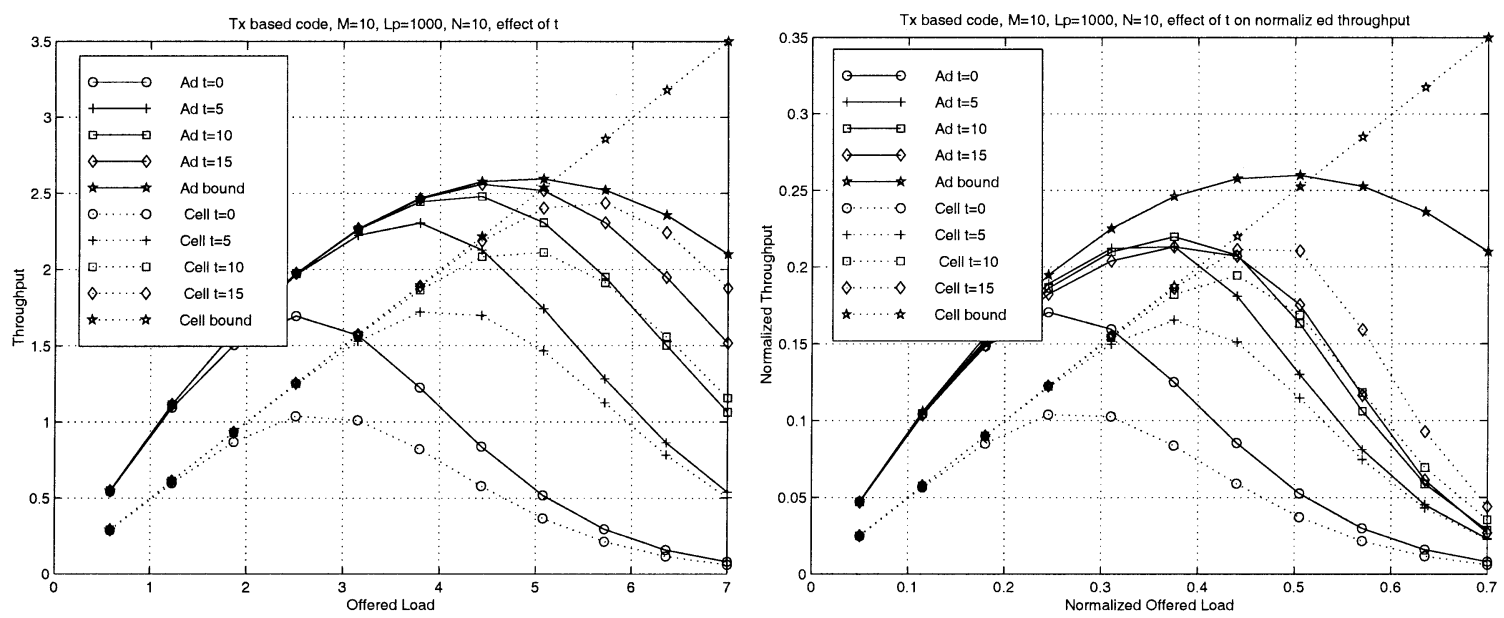

Fig. 6. Throughput versus offered load, $N=10$. Solid line: ad hoc network; dotted line: centrally controlled network; left: actual; right: normalized.
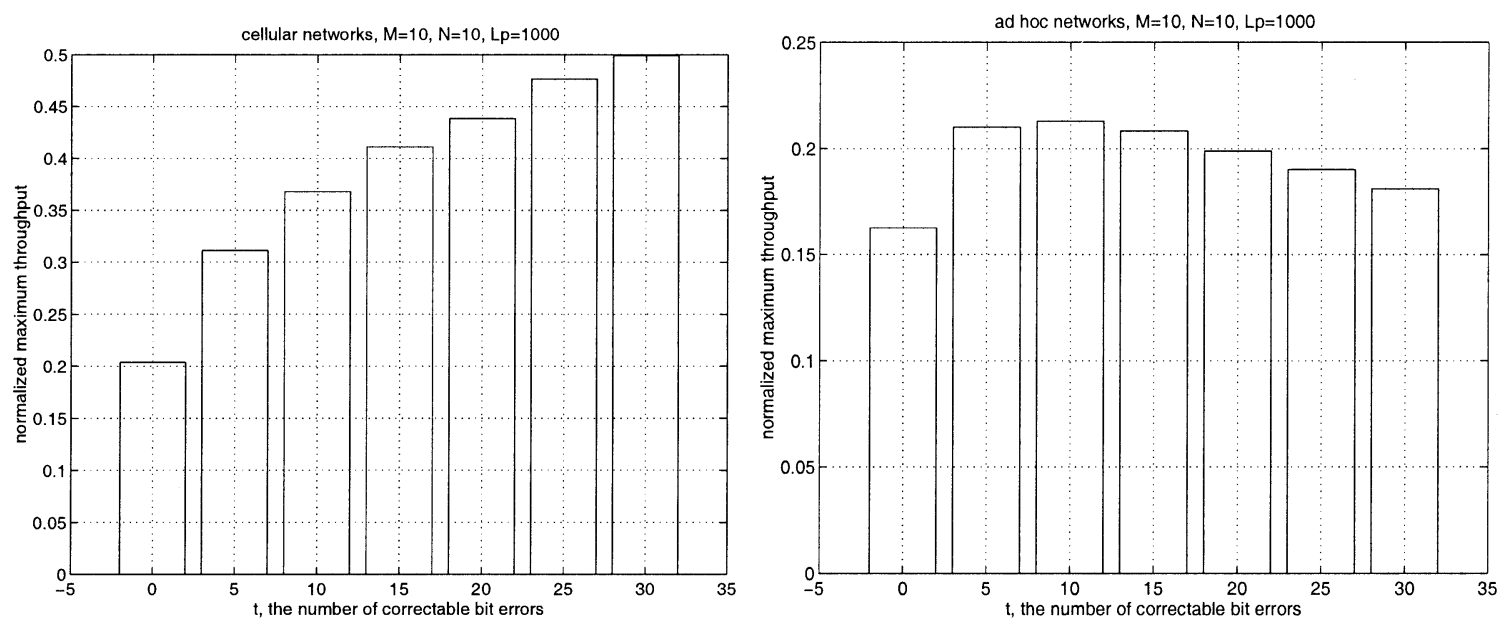

Fig. 7. $\quad t$, the number of correctable bit errors versus maximum normalized throughput.

availability, and the normalized throughput actually decreased with increasing $N$.

Effects of Error Control on the Throughput: Next, we compare the throughput of these two systems with a fixed $N$ at different $t$ and investigate effects of error control on the throughput for both systems. Fig. 6 shows both the actual and the normalized throughput versus offered load for these two systems with $t=0,5,10,15$ at $N=10$. For the centrally controlled system, because the bandwidth expansion caused by larger $t$ is smaller than that caused by increasing $N$, both the normalized and the actual throughput increased as $t$ became larger. Although it is not surprising that more powerful error control will improve the actual throughput in the ad hoc network as shown in the left part of Fig. 6, the relationship between $t$ and normalized throughput turns out to be interesting. As can be observed from the right part of Fig. 6, the normalized throughput increased for all normalized offered load when $t$ was increased from zero to five. But as $t$ was increased from 5 to 15 , larger $t$ did not necessarily lead to higher normalized throughput.

To investigate the relationship between $t$ and the normalized throughput, we plotted the maximum normalized throughput versus $t$ for both systems as shown in Fig. 7 (left: centrally controlled networks, right: ad hoc networks). Clearly, we can see that in centrally controlled networks, larger $t$ leads to higher maximum normalized throughput, but, in the ad hoc network, the maximum normalized throughput is not monotonic. The maximum normalized throughput increased as $t$ was increased from zero to ten. However, for $t>10$, larger $t$ actually leads to decreased maximum normalized throughput. Therefore, a tradeoff can be reached to select a $t$ to maximized the normalized throughput.

\section{Delay-ThroughPut Characteristics COMPARISON}

Denote $W$ as the packet backlogged time, $T$ as the time between the packet arrival and the start of the first available time slot. The delay and the average delay of a (coded) packet in the centrally controlled system are

$$
\begin{aligned}
D_{c} & =W+T+2 \\
\bar{D}_{c} & =E(D)=E(W)+E(T)+2=\bar{W}+1+2 \\
& =\frac{\sum n q_{n}}{\bar{\beta}}+2.5
\end{aligned}
$$

where we assume that a time slot is one unit time and uniformly distributed packet arrival time within a time slot. The last equality follows the approach of Kleinrock and Lam [8]. 

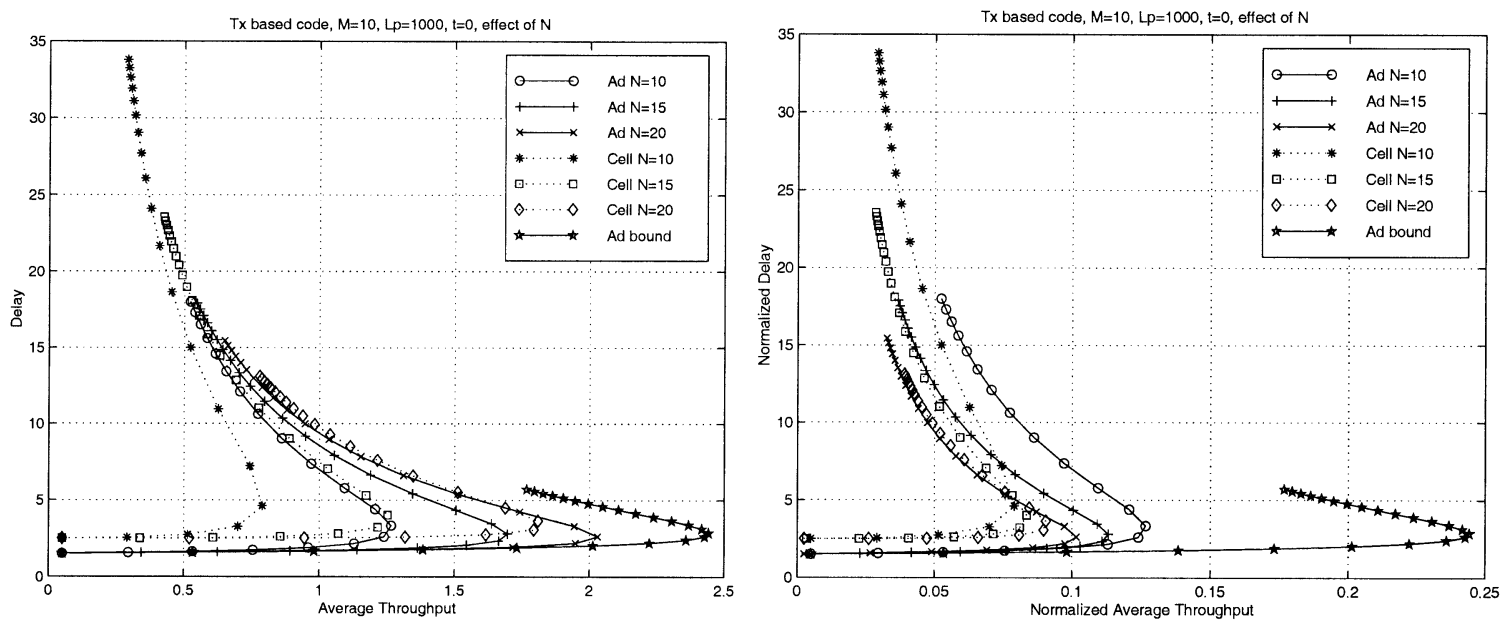

Fig. 8. Delay versus average throughput. Solid line: ad hoc system; dotted line: centrally controlled system; left: actual; right: normalized.
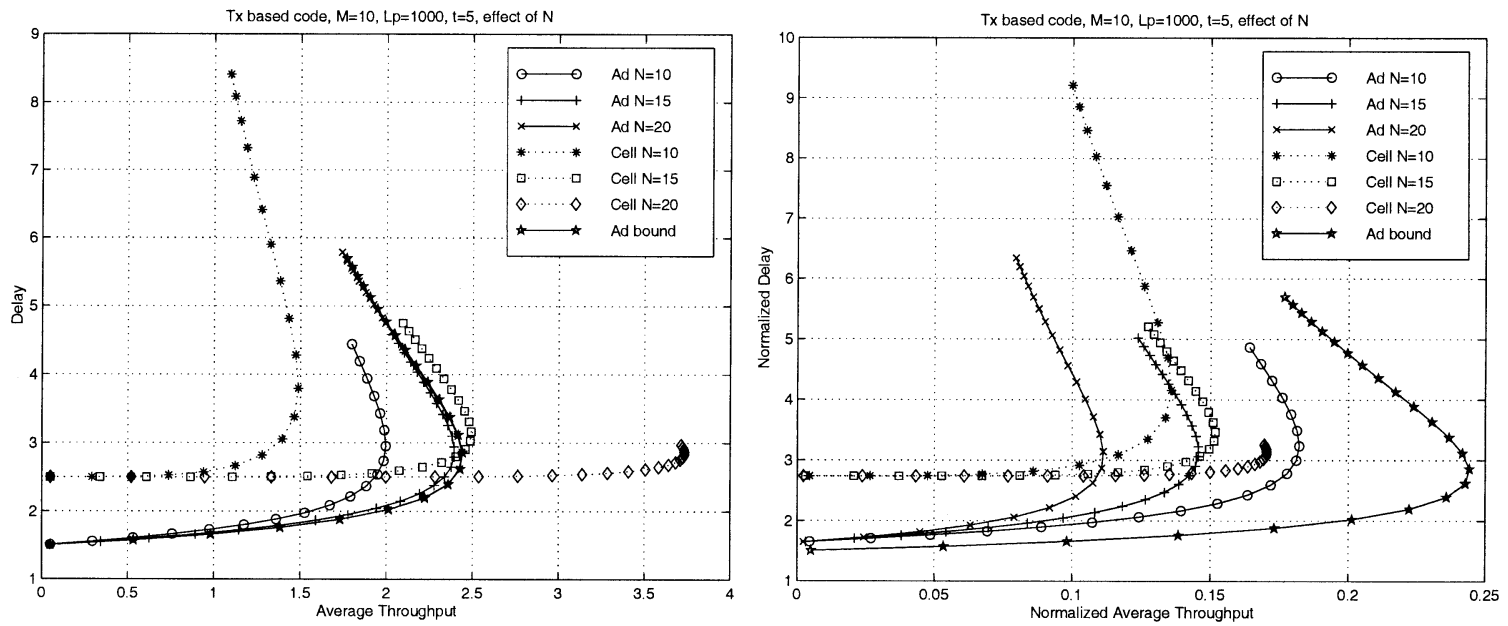

Fig. 9. Delay versus average throughput. Solid line: ad hoc system; dotted line: centrally controlled system; left: actual; right: normalized.

Similarly, we can have the formulae for the delay and average delay for the ad hoc system

$$
\begin{aligned}
D_{a} & =W+T+1 \\
\bar{D}_{a} & =E(D)=E(W)+E(T)+1=\bar{W}+\frac{1}{2}+2 \\
& =\frac{\sum n q_{n}}{\bar{\beta}}+1.5 .
\end{aligned}
$$

Delay Normalization: Using different $t$ values in error control coding introduces different degrees of redundancy into a packet. In order to make a fair comparison among networks using different $t$, we define the the average packet delay of an uncoded packet as the normalized average packet delay (i.e., the summation of the average backlogged time, the transmission time and the average waiting time for the first available time slot of an uncoded packet).

The normalized packet delay can be computed in the following way. From (25) and (26), we can obtain the average delay $\bar{D}$ for a coded packet. For the sake of presentation, we assume the coding rate $r_{c}=m / n$, where $m$ and $n$ are two integers with $m \leq n$. The average delay of $n$ coded packets is $n \bar{D}$. Since $n$ codes packets contain only $m$ uncoded packets, the average delay for an uncoded packet is

$$
\bar{D}_{u}=\frac{n \bar{D}}{m}=\frac{\bar{D}}{\frac{m}{n}}=\frac{\bar{D}}{r_{c}}
$$

which is the same as the normalization of delay used in [10].

\section{A. Delay Versus Average Throughput Evaluation}

Similar to the throughput comparison, we evaluated the delay versus average throughput relation for both the centrally controlled and the ad hoc system at different $N$ and $t$ and studied the effects of spreading gain and error control on delay-throughput characteristics of both systems. $M$ and $L_{p}$ were the same as in the throughput evaluation.

Comparison Between the Centrally Controlled and the Ad Hoc System: Figs. 8 and 9 show the average delay versus average throughput for both systems with a varying $N$ at $t=0,5$. We observed the well-known behavior of the existence of two stable point [8] in both systems: for one average throughput, there are two corresponding delays, one is small (the desired) and the other is large (undesired). This behavior indicates that both the 

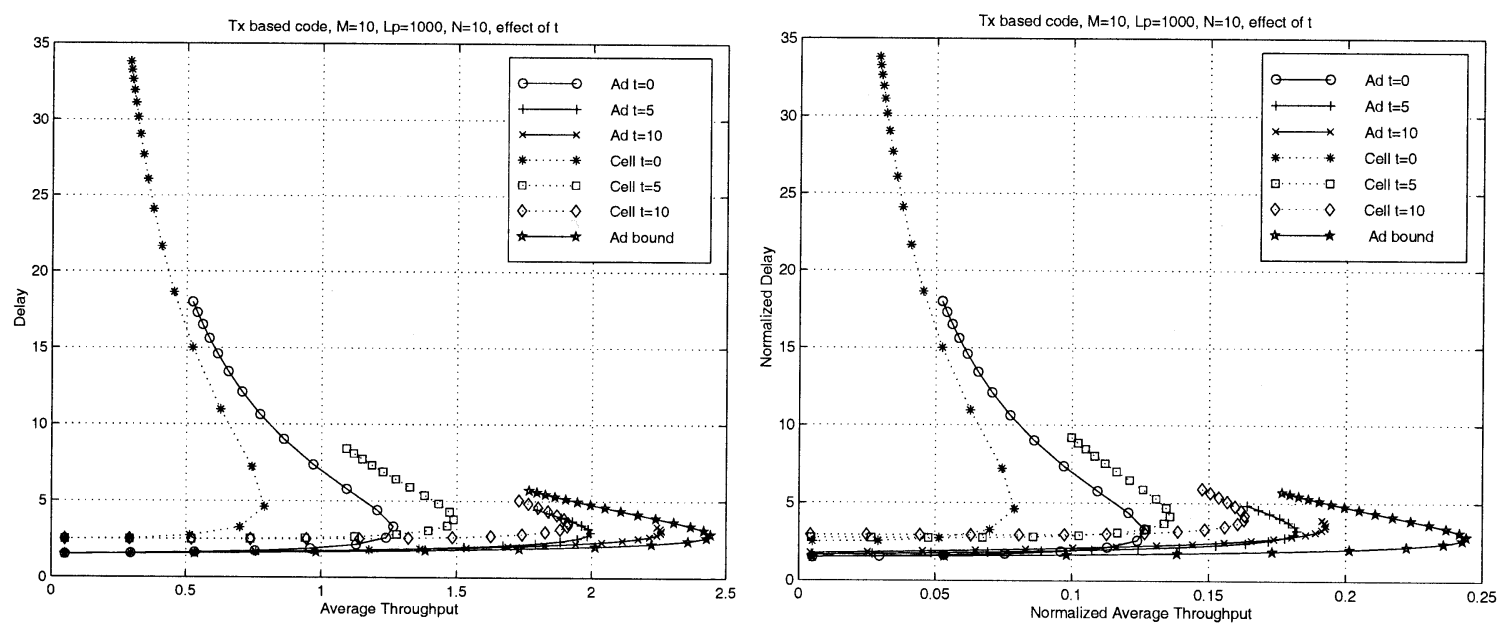

Fig. 10. Delay versus average throughput, $N=10$. Solid line: ad hoc system; dotted line: centrally controlled system; left: actual; right: normalized.

centrally controlled and the ad hoc system are not stable; there is an undesirable stable point of the network state.

The penalty imposed by the centrally controlled architecture on the delay performance can be clearly observed in Figs. 8 and 9. Under light traffic conditions, most of the packet can be received successfully in a time slot. In the ad hoc system, the packet delay was only the transmission and waiting time for the beginning of a time slot. In the centrally controlled system, however, the delay must also includes the downlink transmission time. Therefore, the ad hoc system had smaller packet delay under light traffic conditions. When traffic became heavier, more powerful receivers (larger $N$ and $t$ ) lead to smaller packet delay in the centrally controlled system than in the ad hoc system (see Fig. 9).

Effects of Spreading Gain: $N$ Given the relationship between $N$ and the network throughput observed in Section IV-C, it is not surprising to see that in the centrally controlled network, for a fixed delay value, higher $N$ value increased the normalized average throughput for the centrally controlled network except in networks with poor performance receivers (see the right part of Fig. 8) where larger $N$ decreased the normalized average throughput at the same delay level. In the ad hoc network, larger $N$ uniformly lead to smaller normalized average throughput for the ad hoc system at the same normalized delay.

Effect of Error Control: We also varied $t$ at $N=10$ and evaluated the delay versus average throughput for these two systems as shown in Fig. 10. It is interesting to observe that there is a big gap between the curves with $t>0$ (with error control coding) and the curve corresponding to $t=0$ (no error control coding). This behavior suggests that using error control coding can have significant performance gain in terms of packet delay for both systems.

We included the delay versus average throughput for the perfect receiver (using orthogonal codes with $N=10$ and $t=0$ ) for the ad hoc system as a reference in Fig. 10. We can observe that for the centrally controlled system, when the receiver becomes a perfect receiver, there will be no backlogged time for a packet. As a result, the delay is the summation of the transmission time in the uplink and the downlink and the waiting time for beginning of a time slot. For the ad hoc system, however, even with the perfect receiver, packets still have delay. Larger $t$ value only makes the packet delay performance approaches that of a perfect receiver in the ad hoc network (see the curves corresponding to the perfect receiver and $t=10$ and the ad hoc bound).

The effect of $t$ on the throughput-delay characteristics in the centrally controlled network is the same as $t$ on the throughput. For the ad hoc network, we observed that larger $t$ lead to better throughput-delay behavior for the ad hoc system (higher normalized average throughput at a given normalized delay level).

\section{STABILITY COMPARISON}

It is well-known that slotted Aloha is unstable and has an undesirable stationary point [2]. This behavior is also verified by the delay-throughput characteristics evaluation in the previous section for CDMA slotted Aloha systems. We characterize the stability behavior in terms of FET [8] of the network state. Given a state threshold $n_{c}$, FET $T_{i}$ is the average time the network state first exceeds $n_{c}$ assuming at time zero the network is in state $i$. Following [8], we can obtain $T_{i}$ by solving the following sets of linear equations:

$$
T_{i}=1+\sum_{j=0}^{n_{c}} P_{i, j} T_{j}, \quad i=0,1, \ldots n_{c} .
$$

Note that in the centrally controlled system, the network state changes every two time slots whereas in the ad hoc network the state changes every time slot. Therefore, the FET of the centrally controlled system obtained by (28) has a unit of two time slots.

In evaluating FET, we chose the network state threshold as the state corresponding to the largest throughput for simplicity as is similarly chosen in [4]. Again, we varied $N$ and $t$ to investigate the effect of spreading gain and error control on the FET performance of these two systems. The FET versus normalized arrival rate is illustrated in Figs. 11 and 12 for different $N$ at $t=0,10$.

Comparison Between the Centrally Controlled and the Ad Hoc System: Because the throughput was higher in the ad hoc system than in the centrally controlled system under light traffic 


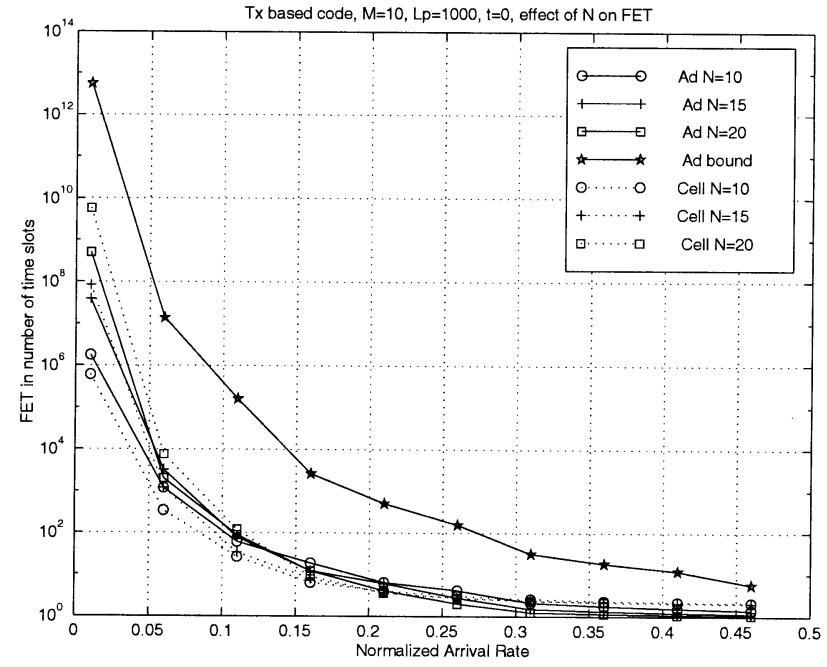

Fig. 11. FET versus normalized arrival rate without error control $(t=0)$. Solid line: ad hoc system; dotted line: centrally controlled system.

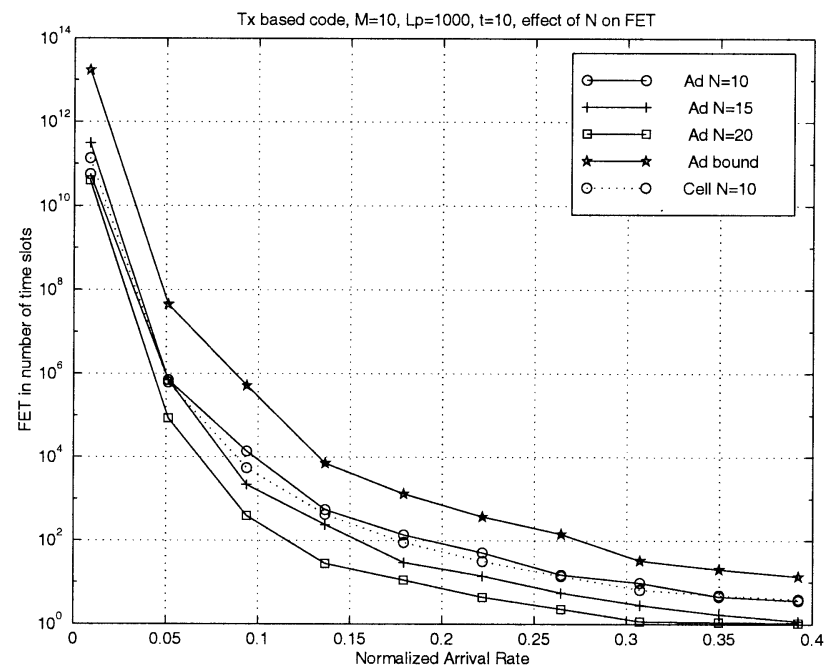

Fig. 12. FET versus normalized arrival rate with error control $(t=10)$. Solid line: ad hoc system; dotted line: centrally controlled system.

conditions, the FET of the ad hoc system was longer than that of the centrally controlled system when the normalized arrival rate was small. As the normalized arrival rate became larger, both systems were saturated and the FET approached one in the ad hoc system and two in the centrally controlled system, respectively. For more powerful receivers $(N=15,20)$, the centrally controlled system had similar FET as that of the ad hoc system under all normalized arrival rate. The reason is two-folded. First, the FET has a unit of two time slots in the centrally controlled system. Second, the offered load (consequently the network state) corresponding to the maximum throughput is higher in the centrally controlled system than in the ad hoc system. Because we define the FET as the time the network state first exceeds the state threshold which maximizes the throughput, the network state in the centrally controlled had to reach a higher threshold than in the ad hoc network, hence, the FET of the two systems could be approximately the same although the ad hoc network had higher throughput than the centrally controlled system under light traffic.
We did not plot FET curves corresponding to $N=15,20$ for the centrally controlled system in Fig. 12 because these FET approached infinity. The reason is that with such a powerful receiver in the centrally controlled system, the throughput almost grew linearly with the arrival rate; hence, the network state never reaches the threshold which corresponds to the maximum throughput. However, in the ad hoc network, the throughput bound made the FET always finite.

Effect of Spreading Gain: In Figs. 11 and 12, we can see that before the network became saturated (FET approached one in the ad hoc network, two in the centrally controlled network), larger $N$ made the network more stable in the centrally controlled system. In the ad hoc system, only under light load (normalized arrival rate less than 0.15 in Fig. 11), did higher $N$ value increase FET at a fixed normalized arrival rate. As the traffic became heavier and before the network saturation, smaller $N$ actually leads to longer FET at a given normalized arrival rate as shown in both Figs. 11 and 12 .

Effect of Error Control Coding: Comparing Figs. 11 and 12 for the same $N$, we can observe that more powerful error control coding $(t=10)$ can greatly increase the FET in the centrally controlled system (for example, with $N=10$ at normalized arrival rate 0.1 , without error control coding, the FET is only about $10^{2}$; but with $t=10$, the FET is $10^{4}$ ). For the ad hoc system at small $N$, error control coding can also greatly improve the FET. For example, at $N=10$, without error control coding $(t=0)$ the system became saturated when normalized throughput was greater than 0.15 . With error control coding, the system reached saturation after normalized arrival rate was greater than 0.3. However, at large $N$, more powerful error control did not affect the FET too much (see the curve corresponding to $N=20$ in Figs. 11 and 12). The reason is again attributed to Theorem 2 because that at larger $N$ most of colliding packets can be recovered by a receiver, it is the ad hoc network throughput bound that limits the throughput. Therefore, increasing $t$ will not affect the throughput too much. Hence, the transition of number of backlogged nodes will not be affect by $t$ significantly.

\section{CONCLUSION}

We have compared the performance of two types of slotted Aloha CDMA wireless LAN: ad hoc and centrally controlled. Based on a finite-population model, the network throughput, the average packet delay and the network FET are derived for both the centrally controlled and the ad hoc system. A performance bound is derived for the ad hoc network. The efficiency of bandwidth utilization is measured by the normalized network performance. The performance comparison between the ad hoc and the centrally controlled networks is evaluated. We also quantitatively analyzed the effect of the spreading gain and the error control coding on the network performance for the two systems.

The performance of the ad hoc network is negatively affected by two factors: 1) availability of a node to receive packets; and 2) the possibility that a node detected packets intended for other nodes. The penalty on the network performance of the ad hoc network caused by these two factors is more significant when the traffic is heavier. Although there is no similar issues in the centrally controlled network, the process of relaying packets by 
the BS penalized the network performance, especially at light traffic conditions. We observed that with the same spreading gain and error control coding, the ad hoc system had higher throughput and smaller packet delay than the centrally controlled system under light traffic conditions; however, the centrally controlled system out performed the ad hoc systems when the traffic was heavy.

For centrally controlled networks, we observed the following.

- For centrally controlled networks with moderate powerful receivers, higher spreading gain can increase normalized network performance.

- When the receiver performance is poor in a centrally controlled network, higher spreading gain actually decreases the normalized network throughput under heavy traffic conditions.

- Because of the smaller bandwidth expansion introduced by the error control than by the spreading gain, it is more efficient to use error control than to use higher spreading gain to improve the centrally controlled network performance.

The effects of spreading gain and error control on the performance of ad hoc networks are more complicated than that of the centrally controlled networks due to the throughput bound imposed by the ad hoc architecture. Specifically, the following observations can be made.

- The improvement of the network throughput obtained from increased spreading gain cannot offset the bandwidth expansion caused by such spreading gain. Increasing spreading gain monotonically decreases normalized throughput; hence, lower efficiency of bandwidth utilization.

- For a given spreading gain, the relationship between the normalized throughput and the number of correctable bit errors is not monotonic. An optimum value of the number of correctable bit errors can be selected to maximize the normalized throughput.

- There is a significant throughput-delay performance improvement from no error control coding to having error control coding both before and after the normalization. As error control coding becomes more powerful, the network reaches the performance bound, hence, the improvement becomes marginal.

- Higher spreading gains improves the network stability at light normalized throughput, but reduces the network stability at heavy normalized throughput.

- Although more powerful coding can increase the network stability for all normalized traffic conditions, the improvement becomes marginal when the spreading gain becomes higher.

\section{APPENDIX A}

\section{A Proof OF THEOREM 1}

Let $\mathcal{N}$ be the random variable for the total number of successfully received packets at the link layer, $\mathcal{L}$ be the random variable for the total number of packets transmitted in a time slot, and $\mathcal{I}$ be the random variable for the total number of packets intended for idle nodes in the network $(\mathcal{L}-\mathcal{I}$ packets are lost due to the use of half-duplex receivers). Then

$$
\begin{aligned}
r_{L n} & =\sum_{l=0}^{L} \operatorname{prob}\{\mathcal{N}=n, \mathcal{I}=l \mid \mathcal{L}=L\} \\
& =\sum_{l=n}^{L} \operatorname{prob}\{\mathcal{N}=n \mid \mathcal{L}=L, \mathcal{I}=l\} q_{L l}
\end{aligned}
$$

where $q_{L l}=\operatorname{prob}\{\mathcal{I}=l \mid \mathcal{L}=L\}$. For the second step above, we have used the fact that only those packets intended for idles nodes have a chance to be detected correctly. Under Assumption 5 , we have

$$
q_{L l}=\left(\begin{array}{c}
L \\
l
\end{array}\right)\left(\frac{M-L}{M-1}\right)^{l}\left(\frac{L-1}{M-1}\right)^{L-l} .
$$

To determine $\operatorname{prob}\{\mathcal{N}=n \mid \mathcal{L}=L, \mathcal{I}=l\}$, we can view the transmission of $l$ packets to $M-L$ idle nodes as the classic problem of assigning $l$ balls randomly to $M-L$ boxes. Each assignment corresponds to a particular transmitting-receiving pattern for the $l$ packets. By solving this problem, we can have

$$
\begin{aligned}
\operatorname{prob}\{\mathcal{N}=n \mid \mathcal{I}=l, \mathcal{L}=l\} \\
=\sum_{J=\min (l, n)}^{\min (l, M-L)} \frac{\left(\begin{array}{c}
M-L \\
J
\end{array}\right)}{(M-L)^{l}} \sum_{\sum_{j=1}^{J} a_{j}=l} \frac{l !}{a_{1} ! a_{2} ! \ldots a_{J} !} \\
\quad \times \sum_{\sum b_{j=1}^{J}=n} \prod_{i=1}^{J} d_{L, a_{i}, b_{j}} .
\end{aligned}
$$

Substituting (31) into (29) yields (12).

Now we need to determine $d_{L, a_{i}, b_{i}}$, the probability that $b_{i}$ out of $a_{i}$ packets intended for the node is received given total $L$ packets are transmitted in a time slot. We again get this probability by solving the following equivalent problem: there are total $L$ balls (total packets in a slot), $a_{i}$ of them are red (intended for this node), the rest of them are white (not intended for this node). One takes out balls (detect packets) without putting them back. Every trial the probability that $k$ balls are taken out is $s_{L k}(k=0,1,2, \ldots L)$ and $d_{L, a_{i}, b_{i}}$ is the probability that exactly $b_{i}$ red balls are taken out out of these $k$ balls ( $b_{i}$ intended packets are detected).

Denote random variable $\mathcal{K}$ as the number of balls taken. In order to take out exactly $b_{i}$ red balls, we must have $b_{i} \leq \mathcal{K} \leq$ $L-\left(a_{i}-b_{i}\right)$. Let random variable $\mathcal{A}$ be the number of red balls are taken out, we have

$$
\begin{aligned}
d_{L, a_{i}, b_{i}} & =\sum_{k=b_{i}}^{L-\left(a_{i}-b_{i}\right)} \operatorname{prob}\left\{\mathcal{A}=b_{i} \mid \mathcal{K}=k\right\} \operatorname{prob}\{\mathcal{K}=k\} \\
& =\sum_{k=b_{i}}^{L-\left(a_{i}-b_{i}\right)} \frac{\left(\begin{array}{l}
a_{i} \\
b_{i}
\end{array}\right)\left(\begin{array}{l}
L-a_{i} \\
k-b_{i}
\end{array}\right)}{\left(\begin{array}{l}
L \\
k
\end{array}\right)} s_{L k}
\end{aligned}
$$

where $\operatorname{prob}\{\mathcal{K}=k\}=s_{L k}$. 


\section{APPENDIX B}

\section{A PROOF OF THEOREM 2}

We prove the theorem in two steps. First, we show that the network throughput is maximized if and only if $r_{L n}=q_{L n}$ which is the probability that $n$ out of $L$ packets are transmitted to idle nodes. Then, we show that if and only if $S=S_{*}$, will we have $r_{L n}=q_{L n}$.

1) Step 1: $\max \beta(n) \Leftrightarrow r_{L n}=q_{L n}$ : The network throughput is give by (20). Because $p_{k}$ is independent of $r_{L n}$, maximizing $\sum_{l=0}^{k} l r_{k l}$ for each $k$ maximizes the network throughput. For a given $k$, denote $\theta=\sum_{l=0}^{k} l r_{k l}$ and for any $r_{k l}$ define

$$
\theta_{0}=\sum_{l=0}^{k} l q_{k l}, \quad \theta_{1}=\sum_{l=0}^{k} l r_{k l} .
$$

We now show that $\theta_{0} \geq \theta_{1}$ with equality holds only when $r_{k l}=$ $q_{k l}$. From (29), we can express $r_{k l}$ as

$$
r_{k l}=\sum_{m=l}^{k} q_{k m} \alpha_{m l}
$$

where $\alpha_{m n}$ is the probability that given $m$ (out of $k$ ) packets intended for idle nodes, $n$ packets in the network are received by their intended receivers. Substituting (34) into (33), we get

$$
\theta_{1}=\sum_{m=0}^{k} q_{k m}\left(\sum_{l=0}^{m} l \alpha_{m l}\right) .
$$

Since $\sum_{l=0}^{m} l \alpha_{m l} \leq m$, therefore, $\theta_{0} \geq \theta_{1}$ with equality holds only when

$$
\alpha_{m l}= \begin{cases}1, & \text { if } l=m \\ 0, & \text { otherwise }\end{cases}
$$

which leads to $r_{k l}=q_{k l}$.

2) Step 2: $r_{L n}=q_{L n}$ If and Only If $S=S_{*}:$ For $S=S_{*}$, we can simplify $d_{L, a_{i}, b_{i}}$ to

$$
d_{L, a_{i}, b_{i}}=\left\{\begin{array}{ll}
0, & \text { if } a_{i} \neq b_{i} \\
1, & \text { if } a_{i}=b_{i}
\end{array} .\right.
$$

Applying (36) to Theorem 1, we have $r_{L n}=q_{L n}$.

Now, we need to show that $r_{k l}=q_{k l}$ implies $S=S_{*}$. For a fixed $k$, from step 1, we know that $r_{k l}=q_{k l}$ if and only if $\alpha_{k k}=$ 1 , i.e., when there are $k$ packets for idle nodes, all of them will be correctly received by their intended receivers with probability 1 . Assume $s_{k k}<1$. Then, if all $k$ packets are all intended for the same idle node, the probability of receiving $k$ packets will be less than 1 , which contradicts $a_{k k}=1$. Therefore, only

$$
s_{i j}=\left\{\begin{array}{ll}
0, & \text { if } j \neq i \\
1, & \text { if } j=i
\end{array} \quad \text { leads to } r_{k l}=q_{k l} .\right.
$$

\section{REFERENCES}

[1] N. Abramson, Multiple Access Communication: Foundations for Emerging Tecnologies. Piscataway, NJ: IEEE Press, 1993.

[2] D. P. Bertsekas and R. Gallager, Data Networks. Englewood Cliffs, NJ: Prentice-Hall, 1992.

[3] D. H. Davis and S. A. Gronemeyer, "Performance of slotted aloha random access with dealy capture and randomlized time of arrival," IEEE Trans. Commun., vol. COM-28, 1980.
[4] P. W. de Graaf and J. S. Lehnert, "Performance comparison of a slotted Aloha DS/SSMA network and a multichannel narrowband slotted Aloha network," IEEE Trans. Commun., vol. 46, pp. 544-551, Apr. 1998.

[5] D. J. Goodman and A. Saleh, "The near/far effect in local Aloha radio communications," IEEE Trans. Veh. Technol., vol. VT-36, pp. 19-27, Feb. 1987.

[6] R. K. Morrow Jr. and J. S. Lehnert, "Bit-to-bit error dependence in slotted DS/SSMA packet systems with random signature sequences," IEEE Trans. Commun., vol. 37, pp. 1052-1061, Oct. 1989.

[7] — - "Packet throughput in slotted Aloha DS/SSMA radio systems with random signature seuences," IEEE Trans. Commun., vol. 40, pp. 1223-1230, July 1992.

[8] L. Kleinrock and S. S. Lam, "Packet switching in a multiaccess broadcase channel: Performance evaluation," IEEE Trans. Commun., vol. COM-23, pp. 410-423, Apr. 1975.

[9] J. Lehnert and M. Pursley, "Error probabilities for binary direct-sequence spread-spectrum communications with random signature sequences," IEEE Trans. Commun., vol. COM-35, pp. 87-98, Jan. 1987.

[10] Z. Liu and M. El Zarki, "Performance analysis of DS-CDMA with slotted aloha random access for packet PCNs," Wireless Networks, vol. 1, pp. 1-16, 1995.

[11] C. Namislo, "Analysis of mobile radio slotted aloha networks," IEEE J. Select. Areas Commun., vol. SAC-2, pp. 583-588, July 1984

[12] A. Polydors and J. Sylvester, "Slotted random access spread-spectrum networks: An analytical framework," IEEE J. Select. Areas Commun., vol. SAC-5, pp. 989-1002, July 1987.

[13] J. Proakis, Digital Communications, 4th ed. New York: McGraw-Hill, 2001.

[14] M. B. Pursley, "Frequency-hop transmission for satellite packet switching and terrestrial packet radio networks," IEEE Trans. Inform. Theory, vol. IT-32, pp. 652-667, Sept. 1986.

[15] _ , "The role of spread-spectrum in packet radio networks," Proc IEEE, vol. 75, pp. 116-134, Jan. 1987.

[16] D. Raychaudhuri, "Performance analysis of random access pakcet-switched code division multiple access systems," IEEE Trans. Commun., vol. COM-29, pp. 895-901, June 1981.

[17] E. Sousa and J. Silvester, "Spreading code protocols for distributed spread-spectrum packet radio networks," IEEE Trans. Commun., vol. 36, pp. 21-29, Mar. 1988.

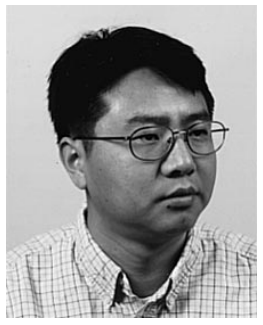

Jeffrey Q. Bao (S'96-M'00) received the B.S and M.S. degrees in electrical engineering, in 1991 and 1994, respectively, from Shanghai Jiao Tong University, Shanghai, China, and the Ph.D. degree in electrical engineering from the University of Connecticut, Storrs, in 1999.

From February 1994 to August 1996, he was a System Engineer with Motorola, Cellular Infrastructure Group, China. From August 1999 to December 2000, he was a DSP Engineer with the Semiconductor Products Sector of Motorola Inc., conducting development on ADSL modems. Since January 2001, he has been with the Broadband Networks Research Lab, Motorola Labs, Mansfield, MA, conducting research and development on various kinds of broadband networks. His research interests include signal processing for communication systems, medium access control in wireless packet networks, and performance analysis of such systems, and quality-of-services issues in broadband networks.

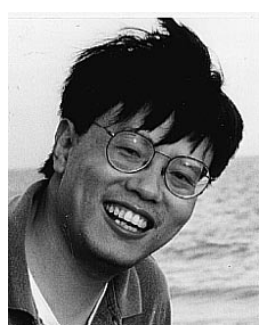

Lang Tong (S'88-M'90-SM'01) received the B.E. degree from Tsinghua University, Beijing, China, in 1985, and the M.S. and Ph.D. degrees in electrical engineering from the University of Notre Dame, Notre Dame, IN, in 1987 and 1990, respectively.

He was a Postdoctoral Research Affiliate, Information Systems Laboratory, Stanford University, Stanford, CA, in 1991. Currently, he is an Associate Professor in the School of Electrical Engineering, Cornell University, Ithaca, NY. Prior to joining Cornell, he was with West Virginia University and later with the University of Connecticut, Storrs. His research interest includes statistical signal processing, adaptive receiver design for communication systems, signal processing for communication networks, and system theory.

Dr. Tong received the Young Investigator Award from the Office of Naval Research in 1996, and the Outstanding Young Author Award from the IEEE Circuits and Systems Society. 